\title{
Tailoring magnetic anisotropy at will in 3D interconnected nanowire networks
}

\author{
Alejandra Ruiz-Clavijo, ${ }^{1}$ Sandra Ruiz-Gómez, ${ }^{2}$ Olga \\ Caballero-Calero, ${ }^{1}$ Lucas Pérez, ${ }^{2,3}$ and Marisol Martín- \\ González ${ }^{1}$ \\ 4 \\ ${ }^{1} I M N$-Instituto de Micro y Nanotecnología, IMN-CNM, CSIC (CEI UAM+CSIC), \\ Isaac Newton, 8, E-28760, Tres Cantos, Madrid, Spain \\ ${ }^{2}$ Dept. Física de Materiales. Universidad Complutense de Madrid. 28040 Madrid, Spain \\ ${ }^{3}$ Instituto Madrileño de Estudios Avanzados - IMDEA Nanociencia, 28049, Madrid, Spain
}

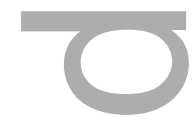

\section{Abstract}

The control of magnetic anisotropy has been the driving force for the development of magnetic applications in a wide range of technological fields from sensing to spintronics. In recent years, the possibility of tailoring the magnetic properties goes together with a need for new 3D materials to expand the applications to a new generation of devices. In this work, we show the possibility of designing the magnetic anisotropy of three-dimensional magnetic nanowire networks just by modifying the geometry of the structure or by composition. We also show that this is possible when the magnetic properties of the structure are governed by the magnetostatic anisotropy. The present approach can guide the systematic tuning of the magnetic easy axis and coercivity in the desired direction at the nanoscale. Importantly, this can be achieved on virtually any magnetic material, alloy or multilayers that can be prepared inside porous alumina. Our results are promising for engineering novel magnetic devices that exploit tailored magnetic anisotropy using metamaterials concept.

This article has been accepted for publication and undergone full peer review but has not been through the copyediting, typesetting, pagination and proofreading process, which may lead to differences between this version and the Version of Record. Please cite this article as doi: $10.1002 / \mathrm{pssr201900263}$ 


\section{INTRODUCTION}

The main properties of the magnetic materials and the most important ones in terms of applications -coercivity, saturation field, domain wall structure, magnetization dynamics... are basically controlled by the magnetic anisotropy ${ }^{1}$. In fact, tailoring the anisotropy has been the driving force of technical and applied magnetism for ages: from the synthesis of magnetoresistive materials for sensing, ${ }^{2}$ to the use of rare-earth elements (with strong magnetocrystalline anisotropy) for the synthesis of strong permanent magnets ${ }^{3}$. When reducing the dimensions, magnetostatic energy (governed by the nanomaterials shape and their saturation magnetization) plays a key role in the global magnetic anisotropy energy ${ }^{4}$. In this sense, nanowires are an excellent playground to understand the material properties since they have a defined magnetization easy axis along the wire axis, determined by magnetostatic (also shape) anisotropy. Recent works have shown exciting magnetic properties of nanowires by controlling the magnetostatic effects: different protected states in the domain wall configuration ${ }^{5-7}$, control in the magnetization processes $^{8,9}$, magnetic ratchet effects ${ }^{10}$, etc. In fact, nanowires have been proposed as building blocks of future magnetic logic devices ${ }^{11}$ and 3D storage devices like the racetrack memories ${ }^{12,13}$.

There is an additional issue for the use of nanowires in practical applications, which is how to manage the nanowires themselves. High-aspect-ratio magnetic nanowire arrays are normally produced by template-assisted deposition ${ }^{14}$. When dissolving the template to work directly with the nanowires, the whole structure collapses into a bunch of individual

nanowires without support, which makes possible to study or use them individually ${ }^{15,16}$ but makes their handling and integration into devices quite complex. Therefore, a further step in the research of magnetic nanowires is to produce structures of interconnected wires at the nanoscale, as proposed for example in the first patent of the race-track memory by $\mathrm{IBM}^{17}$ but never fabricated. Three-dimensional magnetic nanostructures, in fact, can pave the way towards the emergence of new physics andthe development of new devices for their exploitation in numerous applications ${ }^{18}$.

In this work, we present a way to solve all the previous drawbacks, under a singular approach for future novel applications: Three-dimensional (3D) magnetic nano-networks. 
These 3D interconnected magnetic networks combine the advantages of tailoring as desired the magnetic properties at the nanoscale, plus the fact that the 3D linkage network can be handled with tweezers in the same way that it can be done with bulk material, after alumina scaffold removal ${ }^{19,20}$. In other words, when the structure is made of a magnetic material, a free-standing 3D magnetic nano-network is obtained with this process, consisting of an array of vertical nanowires connected periodically each certain distance, $Z$, via transversal nano-connections (like a nano 3D scaffold). This distance $Z$ can be tailored as desired. Therefore, intuitively these kinds of structures could combine the properties of nanowires and films to a certain extent for a single structure of a given material. Moreover, we expect that the shape anisotropy will play an important role in the magnetic behaviour, and taking into account that the fabrication method allows great control over the resulting structure, this should be one of the main features of these 3D magnetic nano-networks.

The fabrication method of such 3D interlinked nanostructures is based on porous alumina membranes filled via electrochemical deposition, which constitutes a low-cost method, higly tunable, with no need of vacuum or atmosphere controlled environment and easily scalable to the industry. Other methods of obtaining 3D nanostructures are ion-track-etched polymeric membranes, however by this technique certain parameters cannot be controlled, such as the exact number and position of interconnections between nanowires ${ }^{21}$, two-photon lithography ${ }^{22}$ or Focused Electron Beam Induced Deposition (FEBID) ${ }^{23}$ (which are comparably much more expensive and timeconsuming). Nevertheless, these methods have the advantage of being able to produce more complex 3D structures of virtually any magnetic material. A review of different fabrication techniques of 3D magnetic nanostructures can be found in reference ${ }^{24}$. It is worth noting that in the case of 3D interconnected nanowires fabricated in ion-track etched membranes, the structure itself is quite different from ours, mainly because the angles formed between the connecting nanowires cannot be, as in our case, parallel and perpendicular to the nanowire axis, and that results in a completely different magnetic behaviour. However, in terms of simplicity, reproducibility and cost, the anodization method is better suited for our study than others. 
The versatility of these 3D alumina membranes has been already proven in other fields, where the precise control over the alumina structure allows to finely tune their intrinsic optical properties, for instance, the optical response of the metamaterial could be fixed to a certain wavelength thus acting as highly sensitive colourimetric sensors ${ }^{25}$. Similarly, the properties of the filling material would be modified. This is the case of a work performed in our group by Resende et al., in which these 3D aluminas were used as templates to produce metamaterials based on polymeric 3D Bragg refractors with a well-controlled optical response ${ }^{26}$. Now, we will translate that same control and tunability over the magnetic response of a magnetic material (having control over the geometry of the structure, the magnetic properties of the metamaterial can be easily controlled and tuned to tailor the desired needs). In this work, the properties of such 3D nano-networks produced from magnetic materials will be studied and considering the geometry influence on the magnetism, one could envisage that these structures could be a tool to finely control the magnetic anisotropy of a given material. And also, the easiness in their implementation inside actual micro and nano-devices to take advantage of their modified properties is another benefit of such 3D magnetic nano-networks.

\section{RESULTS AND DISCUSSION}

\section{A. Ni and Co 3D interconnected nano-networks}

As it is explained in detail in the experimental section, 3D Ni and Co nano-networks with different $Z$ distances between the transversal connections have been fabricated for this work, with $Z=240 \mathrm{~nm}$, and $570 \mathrm{~nm}$, along with the samples consisting of 1D nanowires $(Z=\infty)$ for comparison. Figure 1 shows the Scanning Electron Microscope (SEM) images of 3D nano-networks of interconnected Ni nanowires (NWs). More detailed SEM images of these $3 \mathrm{D}$ nanowire networks for all $Z$ distances can be found in the supporting information (See $\quad$ Figure 
a)

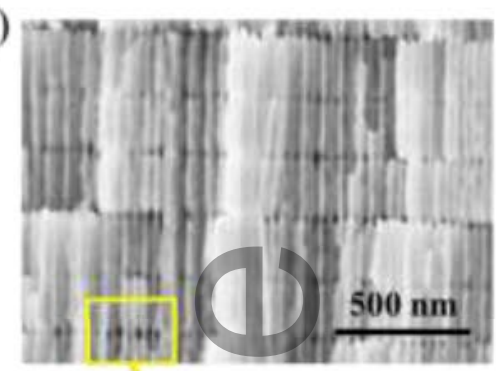

b)
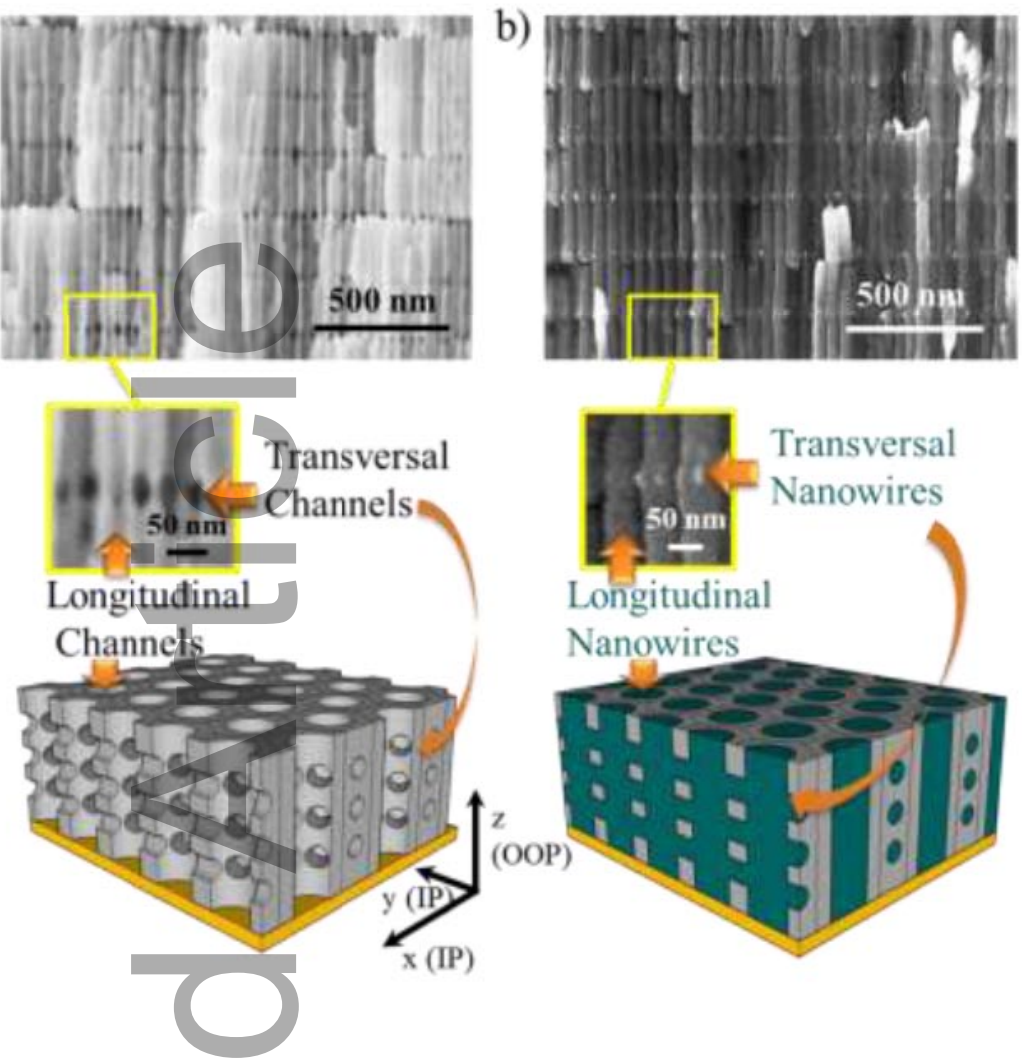

c)
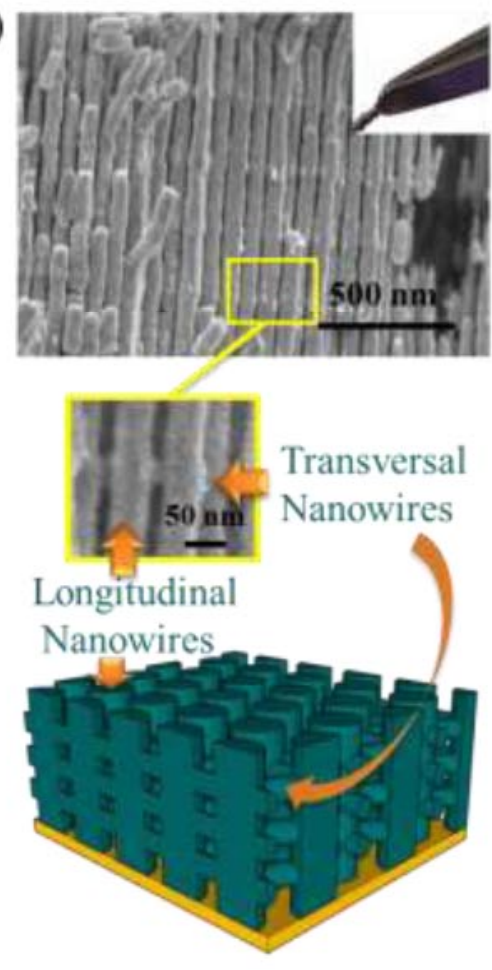

Figure 1. Different images of the fabrication process of a 3D magnetic nanonetwork: a) to c) present both lateral view SEM images and schematics drawings of a) the $3 \mathrm{D}$ alumina matrix b) the alumina matrix filled with $\mathrm{Ni}$, replicating the empty part of the structure, and c) the 3D Ni nano-network once the alumina matrix has been dissolved. The insets show a close up view of the transversal nanochanels through the alumina template and their anti-replica when it is filled, the local tranverse nanointerconnections. The upper inset image in c) presents an optical photo of this freestanding 3D Ni nanowire network hold with a pair of tweezers after alumina matrix removal. The coordinate axis show the different directions along which an external magnetic field is applied during the experiments, in plane (IP) and out of plane (OOP).

X-Ray Diffraction (XRD) analysis was performed in all samples in order to determine their crystalline structure and preferential orientation (See Supporting Information, Figure S2.a). From these measurements, we can conclude that all the 3D Ni nanonetworks were grown with face-centred cubic (fcc) crystalline structure with a clear 
polycrystalline structure. XRD diffraction patterns of Co structures showed a hexagonal closed packed (hcp) polycrystalline structure (shown in Figure S2.b of the Supporting Information). In these spectra, a mean diffraction peak at $2 \theta=41.53^{\circ}$ associated with (100) plane and the second order peak (200) at $2 \theta=90.33^{\circ}$ are observed. Also, a strong (110) peak appears at $2 \theta=75.77^{\circ}$. Therefore, in these samples, the hcp c-axis and, hence, the magneto-crystalline easy axis, is perpendicular to the vertical direction of the nanowires, lying in random orientation but within the plane of the sample. This crystalline structure and orientation were found in Co nano-networks for all $Z$ distances in this work. Also, $\mathrm{Ni}_{1-\mathrm{x}} \mathrm{Co}_{\mathrm{x}}$ alloys with different ratios but the same $Z$ inter-distance have been prepared in this study to observe not only the effect of $Z$ but the effect of composition in the magnetic properties of this 3D networks. All the magnetic measurements that will be shown in this work have been performed with the 3D nano-networks inside the alumina matrix. As the samples are polycrystalline we can assume that the magnetocrystalline and magnetoelastic anisotropies terms of magnetic energy average to zero, all the magnetic properties should be governed by the magnetostatic energy, which is proportional to $M_{S}{ }^{2}$ and strongly depends on the structure shape. If other magnetic components were to influence the preferential easy or hard magnetization axis, apart from the one marked by shape anisotropy, this should be found in the magnetic characterization. Therefore, we proceed with a detailed characterization of the angular dependence of magnetic properties - via the measurements of static hysteresis loops - as a function of geometry and composition. In this work, we defined the out-ofplane (OOP) direction as that along the length of the nanowires and the in-plane (IP) direction as the perpendicular one, that is, parallel to the transversal nano-connections and to the surface of the sample (Figure 1.a).
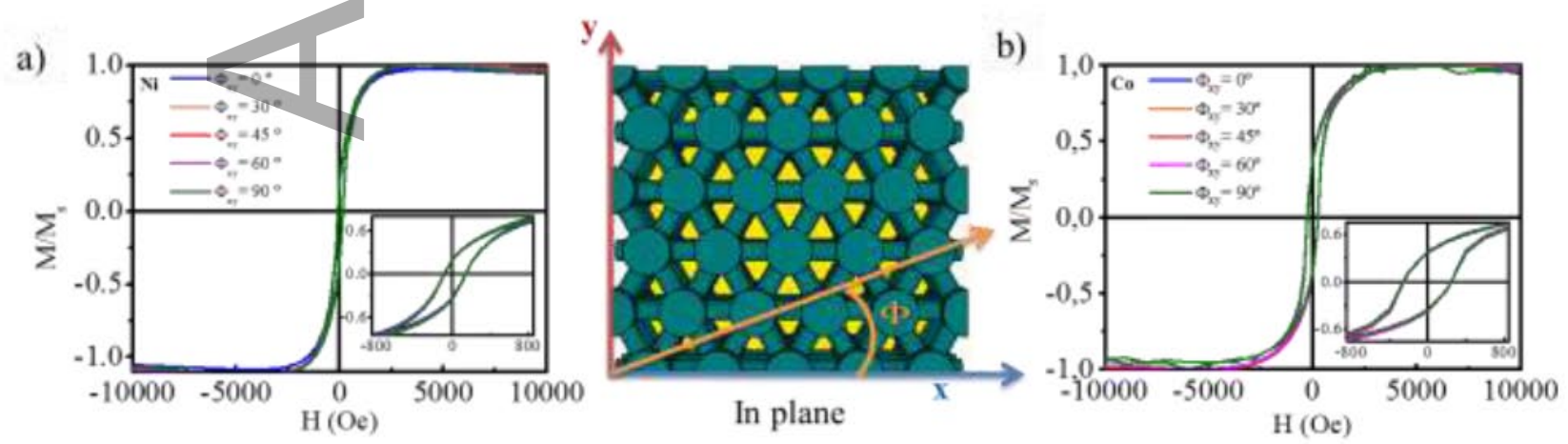

This article is protected by copyright. All rights reserved 
Figure 2. Hysteresis loops measured with the magnetic field applied in different directions along the plane (IP direction) of a) $\mathrm{Ni}$ and b) Co nano-network. The insets show an enlargement of the central part of the hysteresis loops. The drawing in the center represents a schematic top view of a 3D nano-network, corresponding to the xy plane (IP direction) along which the magnetic field was applied during measurements.

First, we investigate the magnetic properties of the different planes forming the 3D nano-network. For this, hysteresis loops were measured with a magnetic field applied inplane (IP) to the sample surface, in different IP directions for both nickel (Figure 2.a) and cobalt (Figure 2.b)3D nano-networks. As can be seen in both figures, the hysteresis loops measured in different directions are the same within the experimental error. The enlargement of the central part of the hysteresis loops shown in the insets of the figure shows that there are also no differences in the low field region. Both $\mathrm{Ni}$ and $\mathrm{Co}$ networks do not show any magnetic anisotropy in the plane. This is somehow expected from the geometry of the nano-networks. Although they are composed by locally ordered hexagonal structures and therefore, there are non-equivalent directions in the plane giving rise to certain anisotropy, the macroscopic samples are composed by randomly distributed oriented regions, also called domains. For this reason, the possible IP magnetic anisotropy contributions average to zero when measuring the macroscopic behaviour of the whole structure. Taking this result into account, in the following we will focus our discussion in analyzing the differences in the magnetic properties measured IP and OOP, assuming that IP means any direction parallel to the plane of the sample and perpendicular to the nanowires and OOP the direction parallel to the NW axis and perpendicular to the sample surface. 

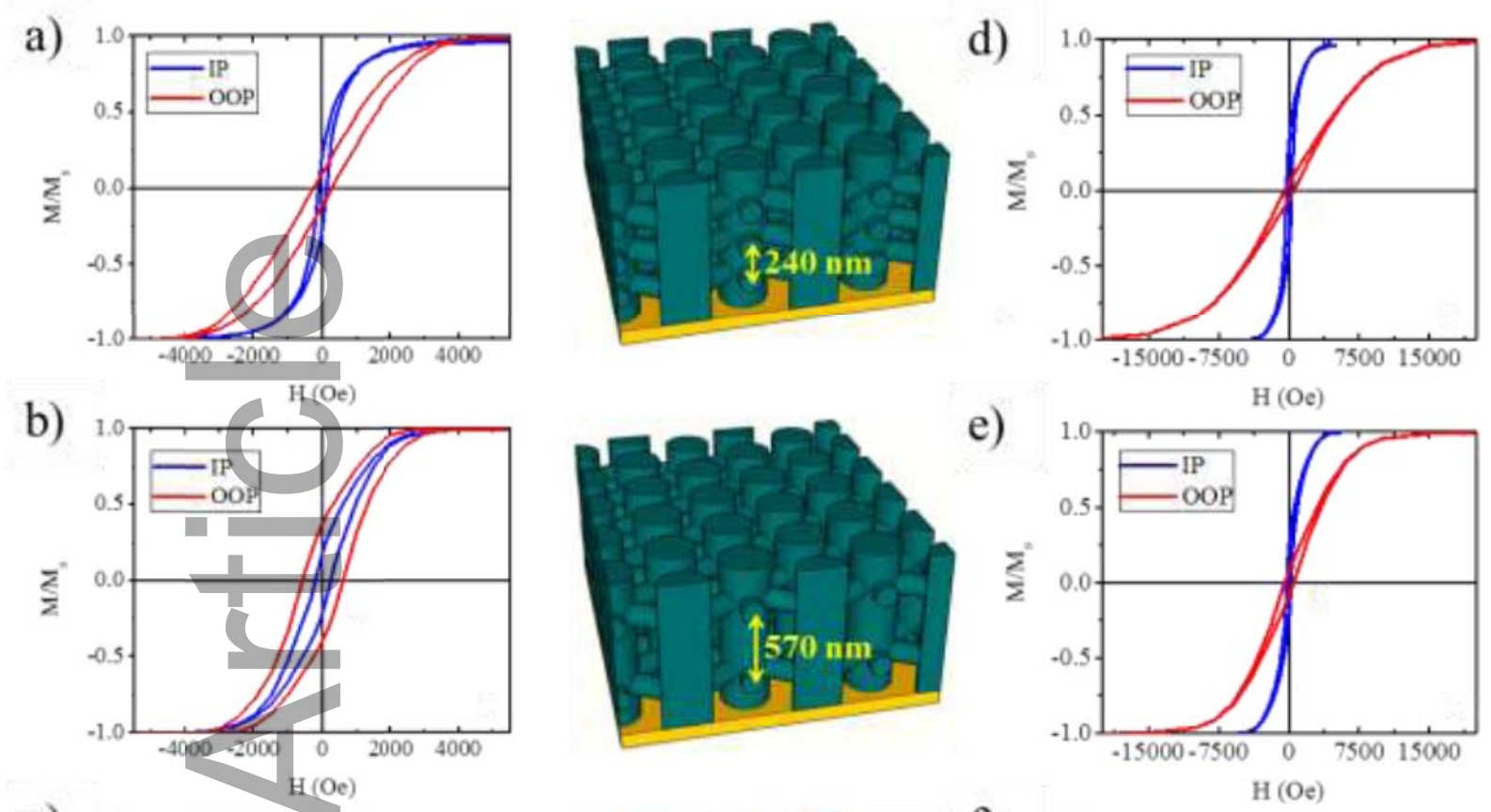

e)

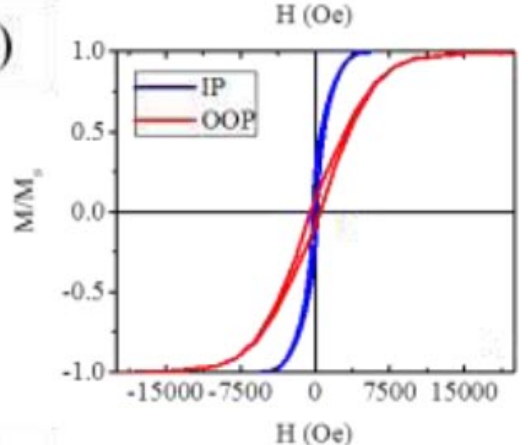

c)
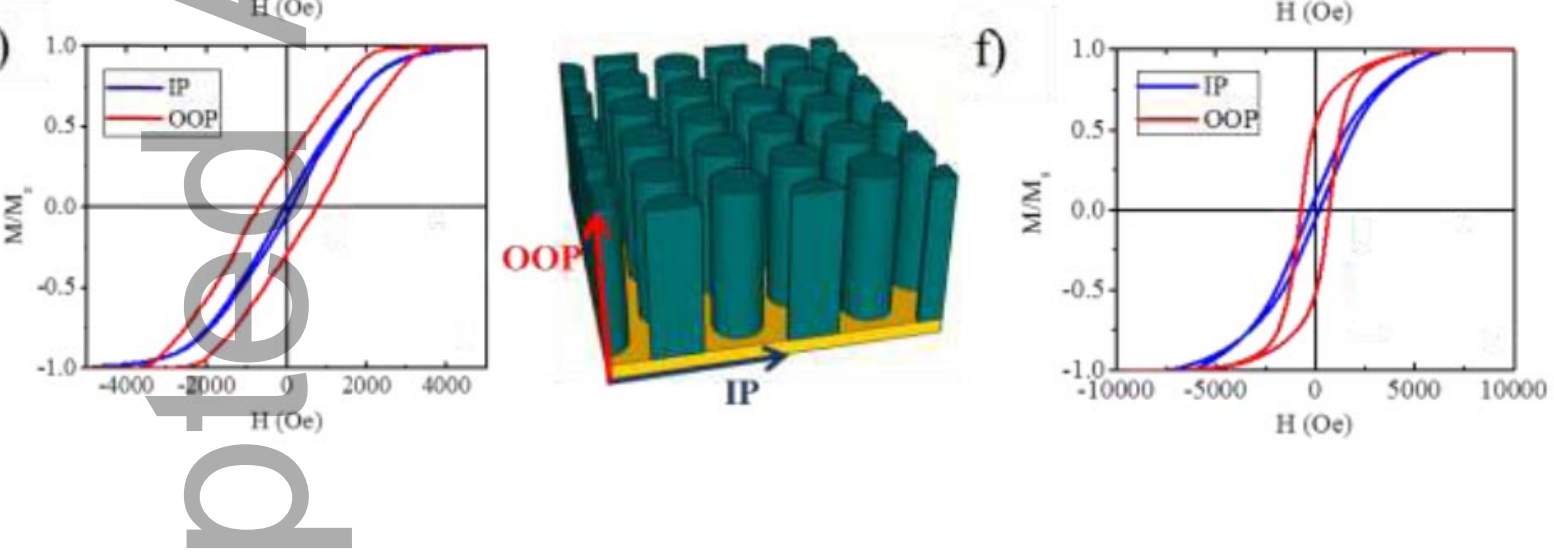

Figure 3. Hysteresis loops measured in 3D Ni Nano-networks being a) $Z=240 \mathrm{~nm}$ and $\quad$ b) $Z=570 \mathrm{~nm}$, and c) $1 \mathrm{D}$ Ni nanowires $(Z=\infty)$. In Co, being d) $Z=240 \mathrm{~nm}$ and e) $Z=570 \mathrm{~nm}$ for 3D Co nano-networks, and f) $Z=\infty$ for $1 \mathrm{D}$ nanowires for 3D Co nano-networks. The central drawings present an squetch of the sample configuration for each case.The IP and OOP axis show the different directions along which an external magnetic field is applied during the experiments.

In Figure 3 we show the hysteresis loops measured in Co and Ni 1D nanowires arrays and nano-networks for two different $Z$ distances. Let us first focus on the behaviour of 3D Ni networks, starting by Figure 3.a. In this case, coercivity is almost zero in the IP direction, pointing out to a magnetization process mainly based on rotation of magnetization, characteristics of a hard magnetization axis. On the other hand, coercivity is larger in the 
OOP direction (parallel to the wire axis), most likely related to nucleation and propagation of the domain walls along the different directions of the structure, typical behaviour of an easy axis. ${ }^{27}$ As the distance between planes is increased (Figure 3.b), the anisotropy increases in the IP direction. This sample becomes almost isotropic. Finally, when increasing further the distance to the infinite, which corresponds to a 1D nanowire array (Figure 3.c), there is an inversion in the direction of the easy axis of magnetization and the OOP direction becomes now the easy axis of the system. This change in the magnetic anisotropy of the sample can be explained using micromagnetic simulations using the OOMMF code, see Supporting Information (Figure S4.b to S4.f). When the distance between planes is small, there is a clear difference between the IP and OOP directions. The easy axis is situated $\mathbb{P}$ whereas the OOP direction (parallel to the NWs axis) is a hard magnetization direction. This behaviour is the opposite than the one observed in the more conventional 1D array of Ni nanowires, where the easy axis is always placed along the nanowire axis, providing the nanowires are not very close. In other words, for the smallest $\mathrm{Z}$ distance, the easy and hard axis are reversed when compared to conventional 1D Ni nanowire arrays. When the distance between planes increases, the system tends to the behaviour of the $1 \mathrm{D}$ nanowire arrays, with an easy axis in the OOP direction. In between, both contributions compensate, and the system becomes isotropic.

As it happened in the case of Ni, for 3D Co nano-networks (Figures 3.d, and 3.e) the magnetization easy axis also change progressively from an IP easy axis to an OOP easy magnetization direction when increasing the $Z$ distance. The reversal of the magnetization is much more pronounced in the case of 3D Cobalt nano-networks, where all the experimental hysteresis loops show a complete reversal compared to that of Co $1 \mathrm{D}$ nanowire arrays $(Z-\infty$, Figure 3.f). This is explained taking into account that the saturation magnetization of Co is three times larger than the one of Ni. Considering that magnetostatic anisotropy scales with $M^{2}$, the contribution of shape anisotropy, for the same geometry, is almost one order of magnitude larger in Co than in $\mathrm{Ni}$, making more difficult for the magnetization to align perpendicular to the planes. The XRD patterns showed a texture in these samples, with the c-axis of the hcp structure parallel to the inplane direction (Figure S2.b). So, an additional contribution from the 
magnetocrystalline anisotropy is also present. Nevertheless, the complete reversal in anisotropy is shown by magnetic simulations in the supporting information (Figure S4.g and S4.h). Micromagnetic simulations showed a switch in the magnetization occurring at a rather large $Z$ value of $3000 \mathrm{~nm}$. In any case, although being more difficult to switch anisotropy in the case of Co, we can conclude that it is also possible. And, that the final orientation of the easy axis is mainly controlled by shape, as in the case of $\mathrm{Ni}$.

\section{B. $\mathrm{Co}_{\mathrm{x}} \mathrm{Ni}_{1-\mathrm{x}} 3 \mathrm{D}$ interconnected nano-networks}

As there is a clear relationship between the magnetization processes and the geometry (when changing the inter-distance $Z$ of the 3D interconnected nano-networks of $\mathrm{Ni}$ and $\mathrm{Co}$ ), one can describe the behaviour using the following equation that takes into account the shape anisotropy $\left(K_{\text {shape }}\right)$ :

$$
\left.K_{\text {shape }}=\frac{1}{2} \mu_{0} N M_{S}^{2} \text { (equation } 1\right)
$$

, where $N$ is the demagnetizing factor, that depends only on the shape of the studied sample, $\mu_{0}$ is the vacuum permeability and $M_{s}$ is the saturation magnetization of the sample.

Considering the clear link between the shape anisotropy and the saturation magnetization, it is interesting to study the behaviour when keeping constant the morphology, but changing the saturation magnetization. For that, we have grown 3D CoNi interconnected networks changing the $\mathrm{Co} / \mathrm{Ni}$ ratio of the alloy in the same $3 \mathrm{D}$ AAO template (with $Z$ of $570 \mathrm{~nm}$ ). In principle, these alloys will have a linear change in the saturation magnetization from the value of Co to the one of $\mathrm{Ni}$.

Hysteresis loops measured for all samples in the IP direction are shown in Figure 4.a. Apart from small differences, due mainly to small misalignment of the sample in the magnetometer, the magnetic behaviour of the samples is the same. The hysteresis loops have been normalized for comparison. However, when measuring in the OOP direction (4.b) a clear difference in the saturation field is observed. The saturation magnetization increases with the Co content in the alloy. When increasing saturation 
magnetization, the anisotropy, which is governed by shape anisotropy, increases, making necessary a larger field to fully saturate the structure.
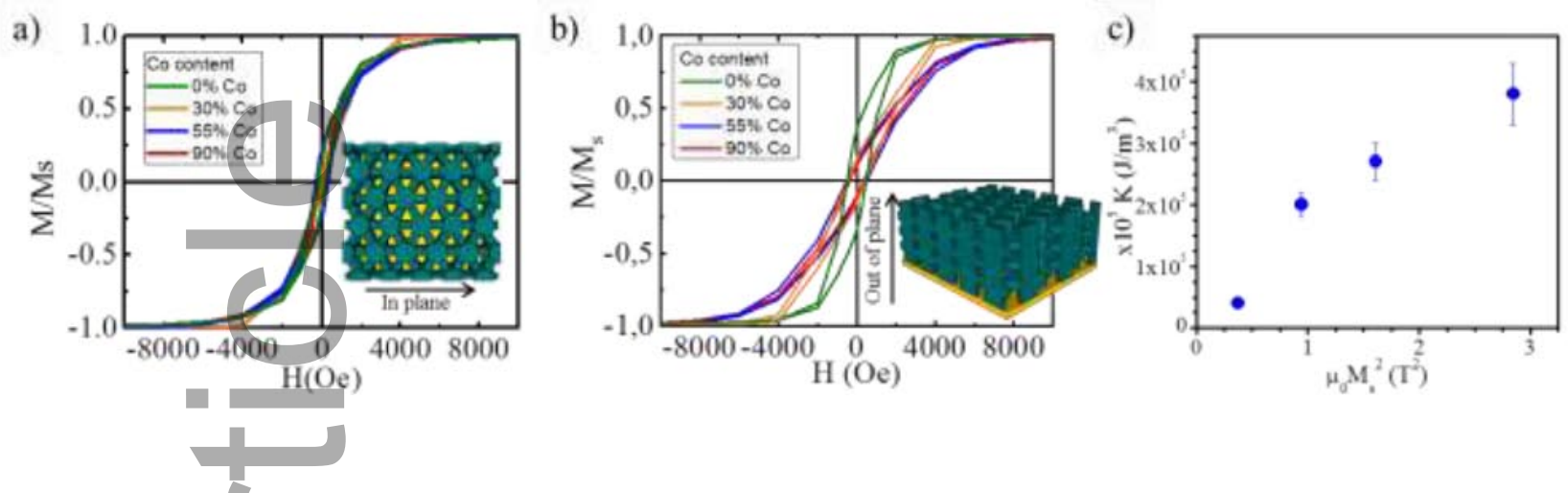

Figure 4. Hysteresis loops measured for different $\mathrm{Co}_{\mathrm{x}} \mathrm{Ni}_{1-\mathrm{x}}$ nano-networks with the applied field in the (a) IP and (b) OOP directions. (c) Anisotropy energy, calculated by numerical integration from the measured first magnetization curves as a function of $\left(\mu_{0} \mathrm{M}_{\mathrm{S}}\right)^{2}$

Anisotropy energy can be calculated by integrating the first magnetization curves, measured after demagnetizing the samples in an oscillating magnetic field and then, increasing the magnetic field in the OOP direction upon saturation. Assuming that shape anisotropy is the main contribution to the total anisotropy energy, from equation 1 , a dependence on $\left(\mu_{0} M_{S}\right)^{2}$ is expected. The calculated values of anisotropy energy have been plotted as a function of $\left(\mu_{0} M_{S}\right)^{2}$ in Figure 4.c, where $M_{s}$ has been estimated from the composition and the values of saturation magnetization of Co and Ni. Although more energy terms should be considered for a better fitting, there is an almost linear relationship between both magnitudes, showing the shape anisotropy is the most important contribution to the total anisotropy energy of these 3D structures, making possible a full control of the anisotropy just by choosing the appropriate composition for the same $Z$ inter-distance. Nevertheless, it is important to remark that this approximation is valid for thin nanowires, where axial configuration of magnetization is expected. For thicker wires, different configuration of magnetic domains, with orthoradial components, would appear, introducing new contributions of the total anisotropy energy ${ }^{28}$. 
This control of the magnetization processes by changing the composition is particularly interesting since recently, it has been shown that changes in composition can lead to full control of magnetization processes in nanowires ${ }^{10,29}$. Even more, the introduction of local changes in composition in nanowires, in which the magnetization processes are fully controlled by magnetostatic interactions, introduces topologically protected magnetic structures, very interesting from both, the theoretical and the application point of view ${ }^{5}$.

The possibility of controlling magnetic anisotropy in 3D structures paves the way for the introduction of these new structures in 3D devices, opening new opportunities for the development of 3D memories, portable magnetic tags, 3D oscillators, etc. In addition, the tunability of our structures, where the magnetization can be finely controlled with composition and by tailoring the interconnection distance, is rather easy to implement. All these without forgetting that is possible to have the 3D interconnected nanowire networks free-standing (without collapsing) and that can be easily handled with tweezers like in a bulk material. Which produce a very interesting material with additional advantages and with the possibility to be applied in macroscopic devices.

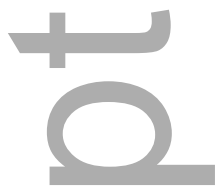

\section{CONCLUSIONS}

In conclusion, we have grown magnetic 3D nanowire interconnected networks, which spacing and composition can be designed to obtain the desired magnetic anisotropy and coercivity. The 3D nanowire network can be described as vertical nanowires interconnected by transversal nanowires connecting each nanowire with its closest neighbours. The interconnections can be spaced along the nanowire length at will. We have demonstrated, that for a given material, we can tune the magnetic properties just by tunning the interconnection distances of the 3D network. Therefore, we can conclude that we have developed a scalable method for fabricating magnetic tunable interconnected 3D nanowire networks, with tunable magnetic properties that can be easily integrated into 
devices thanks to their self-sustained free-standing structure for a wide variety of applications.

\section{EXPERIMENTAL SECTION}

The three-dimensional (3D) structures were fabricated via template-assisted electrochemical deposition. The templates used were anodic aluminium oxide (AAO) produced following the technique described in reference ${ }^{19}$, which consists of a two-steps anodization process in sulfuric acid, a first one to define the order of the nanopores and a second one with a pulsed voltage applied in order to alternate between a mild and a hard anodization along the growth of the length of the pores. Then, the etching of those AAOs in phosphoric acid has different rates for the mild and hard anodized regions and, with careful control over the fabrication parameters, a three-dimensional AAO (3D-AAO) interconnected structure can be produced. This structure consists of nanotubes of around $50 \mathrm{~nm}$ in diameter nanowires separated $65 \mathrm{~nm}$ between them and interconnected by transversal nanochannels of around $40 \mathrm{~nm}$ in height and $30 \mathrm{~nm}$ in diameter. These connecting nanochannels are formed in the areas of hard anodization, and thus the distance between consecutive planes of connecting nanotubes can be finely tuned by changing the pulses in the second anodization step. In such a way, 3D-AAOs with different separation between the planes were fabricated with periodic pulses of three different durations (180 s and $540 \mathrm{~s}$ ) in order to have different separations between the planes of transversal nano-connections (around $240 \mathrm{~nm}$ and $570 \mathrm{~nm}$, respectively). 1D AAOs of the same pore diameter $(50 \mathrm{~nm})$ were also prepared to fabricate the $1 \mathrm{D}$ nanowire arrays for comparison. 


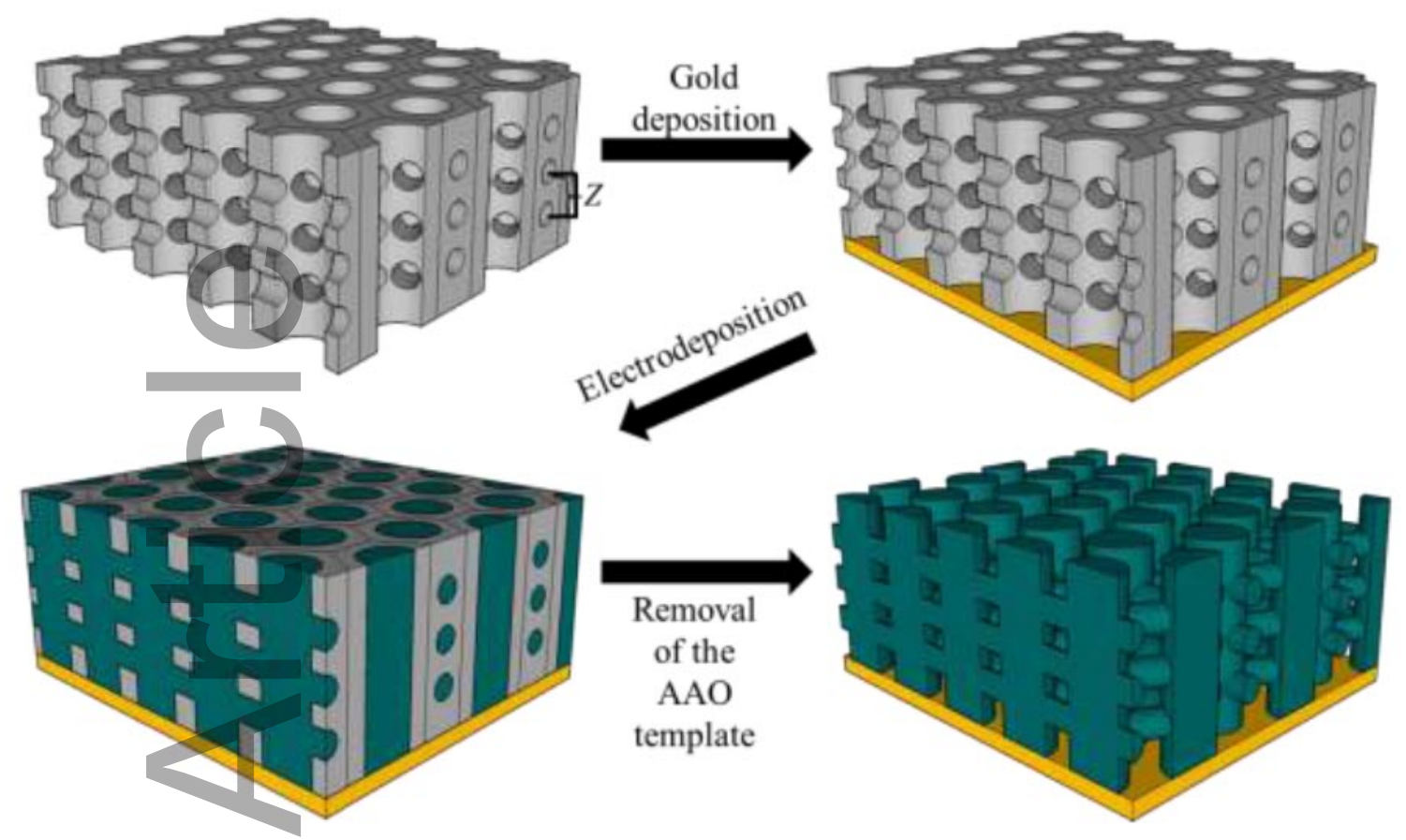

Figure 5. Schematic illustration of the 3D AAO membrane structure as fabricated (top-left), when evaporating a gold layer (top-right), after filling of the membrane with the magnetic material via electrochemical deposition (bottom-left) and once the alumina template is dissolved and the 3D magnetic nano-network is left alone (bottom-right image).. $Z$ refers to the distance between the transversal channels.

These 3D-AAO templates, once the remaining alumina was dissolved, were evaporated with a layer of chromium (5 nm) and gold (150 nm thickness) in one side, and used as working electrodes for a three-electrode electrochemical deposition (with an Autolab potentiostat), with $\mathrm{Ag} / \mathrm{AgCl}$ (saturated $\mathrm{KCl}$ ) reference electrode and a platinum mesh as counter electrode. The electrochemical baths used were $0.75 \mathrm{M} \mathrm{NiSO}_{4} \cdot 6 \mathrm{H}_{2} \mathrm{O}$, $0.02 \mathrm{M} \mathrm{NiCl}_{2} \cdot 6 \mathrm{H}_{2} \mathrm{O}, 0.4 \mathrm{M} \mathrm{H}_{3} \mathrm{BO}_{3}$ and $0.016 \mathrm{M}$ of saccharine for nickel deposition, $0.1 \mathrm{M} \mathrm{CoSO}_{4} \cdot 7 \mathrm{H}_{2} \mathrm{O}$, and $0.4 \mathrm{M} \mathrm{H}_{3} \mathrm{BO}_{3}$ for cobalt deposition. To grow $\mathrm{Co}_{\mathrm{x}} \mathrm{Ni}_{1-\mathrm{x}}$ alloys, both electrolytes were mixed in different ratios to obtain different compositions in the alloy. In all cases, cyclic voltammetry was performed and then the optimal potential vs $\mathrm{Ag} / \mathrm{AgCl}$ reference electrode was chosen, resulting of $-0.9 \mathrm{~V}$ vs $\mathrm{Ag} / \mathrm{AgCl}$ at $45^{\circ} \mathrm{C}$ in the case of nickel and $-0.8 \mathrm{~V}$ vs $\mathrm{Ag} / \mathrm{AgCl}$ at room temperature for cobalt. The actual parameters of the deposit had to be finely tuned in order to obtain a homogeneous growth front with a high filling ratio. This was achieved through pulsed depositions with 
pulses alternating between the desired applied potential for 1 second and time off of 0.1 seconds (with no current applied). In such a way, both 3D cobalt and nickel nanonetworks were grown with the 2 different $Z$ distances between the transversal nanoconnections. A schematic illustration of the empty 3D AAO membrane and of the final 3D magnetic nano-network is presented in Figure 5, where the inter-pore distance between transversal nano-connections is defined as $Z$. $1 \mathrm{D} \mathrm{Ni}$ and Co nanowires were grown under the same conditions described above.

All the deposited nanostructures were studied under a scanning electron microscope (SEM) (Verios 460 from FEI) to verify that the connecting nanotubes were also filled and that we effectively had replicated the 3D-AAO structure, as it was shown in Figure 1 and Figure S1. Also, their structure was analyzed via X-Ray Diffraction spectra (XRD Philips X'Pert four circles diffractometer).

A 7304 LakeShore vibrating sample magnetometer (VSM) allowed us to study the magnetic behaviour of the 3D structures at room temperature. Magnetic hysteresis loops were measured using a magnetic field of up to $10000 \mathrm{Oe}(1 \mathrm{~T})$. The samples were rotated in the field to measure the magnetization processes with the applied field parallel to the plane (IP $-\mathrm{x}$-axis) and along the wires, i.e. out of plane (OOP $-\mathrm{z}$-axis). The magnetic behaviour of the 3D nano-networks has been simulated using the Object Oriented MicroMagnetic Framework (OOMMF) code $^{30}$. We have simulated the magnetization processes as a function of the geometry of the structure, which we have simplified to an array of nanowires with perpendicular planes (see Supporting information, Figure S3). Although different numbers of wires and planes have been simulated, the results were analogous, and all the reported results in this work correspond to structures made by seven nanowires and three planes. The separation between planes $(Z)$ has been modulated from $10 \mathrm{~nm}$ to $500 \mathrm{~nm}$ in the case of nickel and up to $3000 \mathrm{~nm}$ in the case of cobalt. The size of the discretization grid was chosen to be $4 \mathrm{~nm}$ and no thermal effect was included in the simulations.

\section{ACKNOWLEDGMENTS}

This article is protected by copyright. All rights reserved 
The authors acknowledge financial support from INFANTE 201550E072, and MAT2017-86450-C4-3-R and MAT2017-87072-C4-2-P. We also acknowledge the service from the X-SEM Laboratory at IMM, and funding from MINECO under project CSIC13-4E-1794 with support from EU (FEDER, FSE). The authors would also like to acknowledge the help of Mr. Pedro Campos with the sketch of Figure 5.

1 J. M. D. Coey, Magnetism and Magnetic Materials (Cambridge University Press, 2010).

2 P. Perna, D. Maccariello, F. Ajejas, R. Guerrero, L. Méchin, S. Flament, J. Santamaria, R. Miranda, and J. Camarero, Adv. Func. Mater. 27, 1700664 (2017).

3 N. Jones, Nature 472, 22 (2011).

4 J. Sellmyer and R. Skomski, Advanced Magnetic Nanostructures (Springer Science \& Business Media, 2016).

5 S. Ruiz-Gómez, M. Foerster, L. Aballe, M. P. Proenca, I. Lucas, J. L. Prieto, A. Mascaraque, J. de la Figuera, A. Quesada, and L. Pérez, Sci. Rep. 8, 16695 (2018).

6 J. A. Fernandez-Roldan, R. Perez del Real, C. Bran, M. Vazquez, and O. ChubykaloFesenko, Nanoscale 10, 5923 (2018).

7 S. D. Col, S. Jamet, N. Rougemaille, A. Locatelli, T. O. Mentes, B. S. Burgos, R. Afid, M. Darques, L. Cagnon, J. C. Toussaint, , and O. Fruchart, Phys. Rev. B 89, 180405 (2014).

8 Y. P. Ivanov, A. Chuvilin, S. Lopatin, and J. Kosel, ACS nano 10, 5326 (2016).

9 S. D. Col, S. Jamet, M. Stano, B. Trapp, S. L. Denmat, L. Cagnon, J. C. Toussaint, and O. Fruchart, Appl. Phys. Lett. 109, 062406 (2016).

10 C. Bran, E. Berganza, J. A. Fernandez-Roldan, E. M. Palmero, J. Meier, E. Calle, M. Jaafar, M. Foerster, L. Aballe, A. F. Rodriguez, R. P. del Real, A. Asenjo, O. Chubykalo-Fesenko, and M. Vazquez, ACS Nano 12, 5932 (2018).

11 D. A. Allwood, G. Xiong, C. C. Faulkner, D. Atkinson, D. Petit, and R. P. Cowburn, Science 309, 1688 (2005).

12 S. S. P. Parkin, M. Hayashi, and L. Thomas, Science 320, 194 (2008).

13 S. S. P. Parkin and S. H. Yang, Nat. Nanotechnol. 10, 195 (2015). 
14 C. T. Sousa, D. C. Leitao, M. P. Proenca, J. Ventura, A. M. Pereira, and J. P. Araujo, Appl. Phys. Rev. 1, 031102 (2014).

15 B. Ozkale, N. Shamsudhin, G. Chatzipirpiridis, M. Hoop, F. Gramm, X. Chen, X. Marti, J.Sort, E. Pellicer, and S. Pane, ACS. App. Mater. interfaces 7, 7389 (2015).

16 A. I. Mart'inez-Banderas, A. Aires, F. J. Teran, J. E. Perez, J. F. Cadenas, N. Alsharif, T. Ravasi, A. L. Cortajarena, and J. Kosel, Sci. Rep. 6, 35786 (2016).

17 S. S. P. Parkin, "Shiftable magnetic shift register and method of using the same," (2005), US Patent 6,834,005.

18 A. Fernandez-Pacheco, R. Streubel, O. Fruchart, R. Hertel, P. Fischer, and R. P. Cowburn, Nat. Commun 8, 15756 (2017).

19 J. Martín, M. Martín-González, J. F. Fernández, and O. Caballero-Calero, Nat. Commun. 5, 5130 (2014).

20 A. Ruiz-Clavijo, O. Caballero-Calero, and M. Martín-González, Nanomaterials 8, 345 (2018).

21 E. Araujo, A. Encinas, Y. Velázquez-Galván, J. M. Mart́nez-Huerta, G. Hamoir, E. Ferain, and L. Piraux, Nanoscale 7, 1485 (2015).

22 G. Williams, M. Hunt, B. Boehm, A. May, M. Taverne, D. Ho, S. Giblin, D. Read, J. Rarity, R. Allenspach, and S. Ladak, Nano Research 11, 845 (2018).

${ }^{23}$ L. Keller, A. Mamoori, M. K. Pieper, J. Gspan, C. Stockem, I. Schröder, J. Müller, Scientific Reports 8, 1, 6160 (2018)

${ }^{24}$ O. Caballero-Calero, M. Martín González, Chapter in Micro and Nanomagnetic wires, editor M. Vázquez, Elsevier, to be published.

${ }^{25}$ A. Ruiz-Clavijo, Y. Tsurimaki, O. Caballero-Calero, G. Ni, G. Chen, S. V. Boriskina, and M. Martín-González, ACS Photonics 5, 2120 (2018).

${ }^{26}$ P. M. Resende, R. Sanz, O. Caballero-Calero, and M. Martín-González, Advanced Optical Materials 6, 1870082 (2018).

${ }^{27}$ M. Vázquez, K. Pirota, M. Hernandez-Velez, V.M. Prida, D. Navas, R.Sanz, J. Velazquez, Journal of Applied Physics 95 11, 6642 (2004)

${ }^{28}$ C. Bran, J. A. Fernandez-Roldan, E. M. Palmero, E. Berganza, J. Guzman, R. P. del Real, A. Asenjo, A. Fraile Rodríguez, M. Foerster, L. Aballe, O. Chubykalo-Fesenko, and M. 
Vazquez. Phys. Rev. B 96 (2017) 125415

${ }^{29}$ C. Bran, Y. P. Ivanov, J. Kosel, O. Chubykalo-Fesenko, and M. Vazqueź, Nanotechnol. 28, 095709 (2017).

${ }^{30}$ M. Donahue and D. Porter, OOMMF User's Guide, Version 1.0, Tech. Rep. Interagency

Report NISTIR 6376 (National Institute of Standards and Technology, Gaithersburg, MD, 1999).

Captions
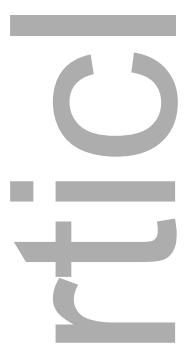

FIG. 1. Shows different images of the fabrication process of a 3D magnetic nano-network: a) to c) present SEM images of a lateral view of a) the 3D alumina matrix b) the alumina matrix filled with $\mathrm{Ni}$, replicating the empty part of the structure, and c) the 3D Ni nano-network once the alumina matrix has been dissolved. Inset image presents an optical photo of this freestanding 3D Ni nano-network hold with a pair of tweezers without the alumina matrix.

FIG. 2. Hysteresis loops measured with the magnetic field applied in different directions along the plane (IP direction) of a) $\mathrm{Ni}$ and b) Co nano-network.

FIG. 3. Hysteresis loops measured in Ni nano-networks being a) $1 \mathrm{D}$ nanowires, b) $Z=570 \mathrm{~nm}$, and c) $Z=240 \mathrm{~nm}$ and in Co nano-networks, being d) $1 D$ nanowires, e) $Z=570 \mathrm{~nm}$ and f) $Z=$ $240 \mathrm{~nm}$.

FIG. 4 Hysteresis loops measured for different CoxNi1-x nano-networks with applied field in the (a) IP and (b) OOP directions. (c) evolutions of the anisotropy as a function of the Co content.

FIG. 5. Schematic illustration of the 3D AAO membrane structure as fabricated (top-left), when evaporating a gold layer (top-right), after filling of the membrane with the magnetic material via electrochemical deposition (bottom-left) and once the alumina template is dissolved and the 3D magnetic nano-network is left alone (bottom-right image). $\mathrm{Z}$ refers to the distance between the transversal channels. 
3D interconnected magnetic nano-networks are grown with tailored anisotropy by controlling their geometry or their composition. In these nano-structures the magnetic properties are governed by the magnetostatic anisotropy, enabling to tune the magnetic easy axis and coercivity in the desired direction through a simple and low-cost fabrication method based on tridimensional porous alumina membranes filled with magnetic materials by electrodeposition.
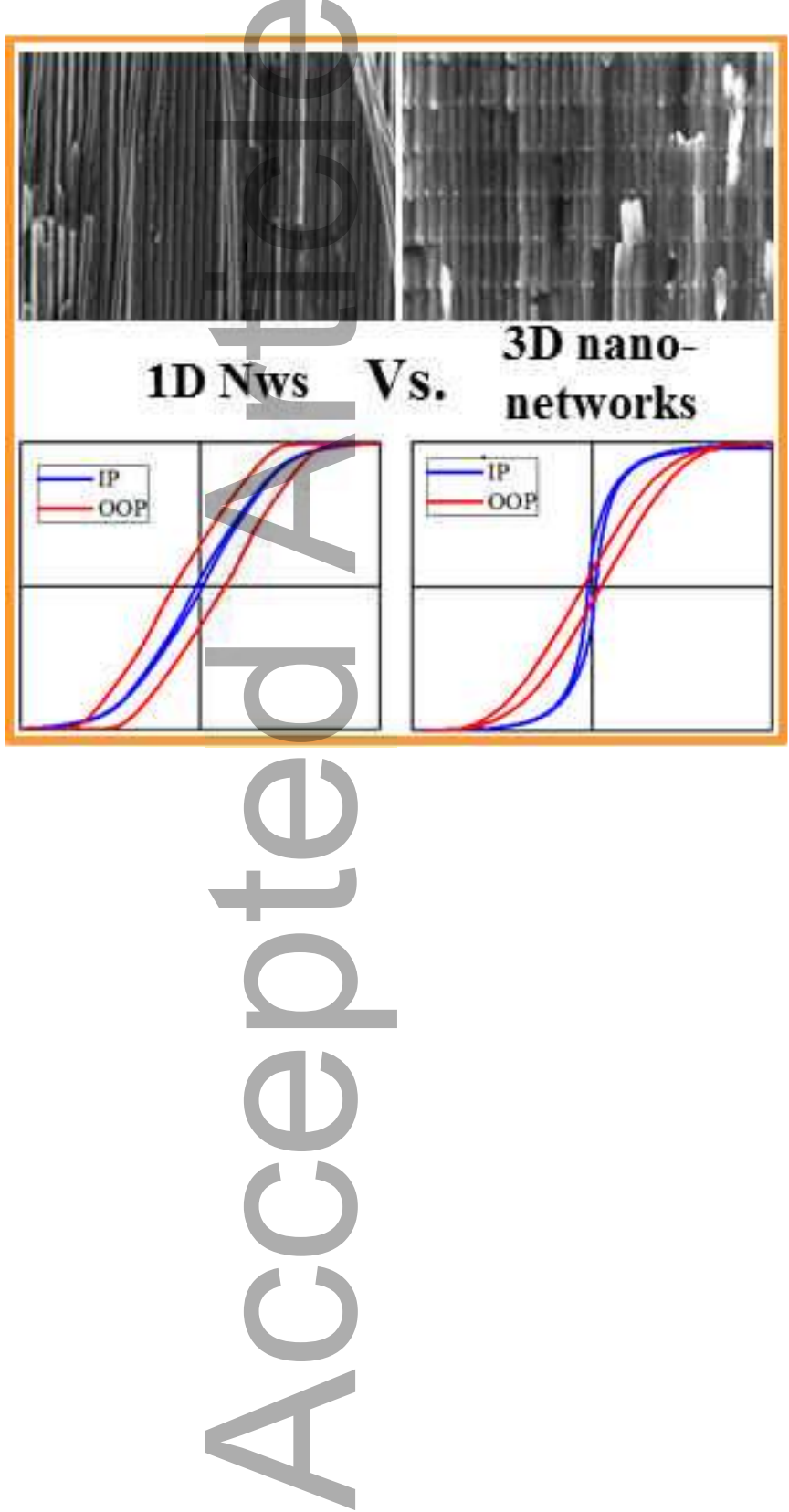

This article is protected by copyright. All rights reserved 